\title{
NUMERICAL ASSESSMENT OF TWO-CHAMBER MUFFLERS WITH PERFORATED PLUG/NON-PLUG TUBES UNDER SPACE AND BACK PRESSURE CONSTRAINTS USING SIMULATED ANNEALING
}

Min-Chie Chiu

Department of Automatic Control Engineering, ChungChou Institute of Technology, Changhua County, Taiwan, R.O.C., minchie.chiu@msa.hinet.net

Ying-Chun Chang

Department of Mechanical Engineering, Tatung University, Taipei, Taiwan, R.O.C.

Follow this and additional works at: https://jmstt.ntou.edu.tw/journal

Part of the Engineering Commons

\section{Recommended Citation}

Chiu, Min-Chie and Chang, Ying-Chun (2011) "NUMERICAL ASSESSMENT OF TWO-CHAMBER MUFFLERS WITH PERFORATED PLUG/NON-PLUG TUBES UNDER SPACE AND BACK PRESSURE CONSTRAINTS USING SIMULATED ANNEALING," Journal of Marine Science and Technology: Vol. 19: Iss. 2, Article 10.

DOI: $10.51400 / 2709-6998.2153$

Available at: https://jmstt.ntou.edu.tw/journal/vol19/iss2/10

This Research Article is brought to you for free and open access by Journal of Marine Science and Technology. It has been accepted for inclusion in Journal of Marine Science and Technology by an authorized editor of Journal of Marine Science and Technology. 


\title{
NUMERICAL ASSESSMENT OF TWO-CHAMBER MUFFLERS WITH PERFORATED PLUG/NON-PLUG TUBES UNDER SPACE AND BACK PRESSURE CONSTRAINTS USING SIMULATED ANNEALING
}

\author{
Min-Chie Chiu* and Ying-Chun Chang**
}

Key words: numerical decoupling technique, space constraints, back pressure constraints, $S A$ optimization.

\section{ABSTRACT}

Recently, research on new techniques of single-chamber mufflers equipped with perforated tubes has been addressed. However, research work on shape optimization of multichamber silencers hybridized with perforated plug/non-plug tubes along with work on the maximal allowable back pressure of mufflers has been neglected. Therefore, we will not only analyze the sound transmission loss (STL) of a spaceconstrained two-chamber muffler hybridized with perforated plug/non-plug tubes but also optimize the best design shape under a specified pressure drop. In this paper, both the numerical decoupling technique and simulated annealing $(S A)$ for solving the coupled acoustical problem of perforated plug/ non-plug tubes are used. A numerical case in eliminating a broadband fan noise is also introduced. To verify the reliability of $S A$ optimization, optimal noise abatements for the pure tone $(500 \mathrm{~Hz})$ are exemplified. However, before the $S A$ operation can be carried out, the accuracy of the mathematical model must be checked using the experimental data. Results indicate that the maximum STL is precisely located at the desired target tones. The optimal result of one case study for eliminating broadband noise also revealed that the overall noise reductions with respect to the muffler, which are under various maximum allowable pressure drops $(100,200,300$, 400, $500(\mathrm{~Pa})$, and infinity) can reach 68.4, 52.7, 45.4, 40.4, 36.0 , and $33.2 \mathrm{~dB}$. Furthermore, both the pressure drop and the acoustical performance increase when the diameters (at

Paper submitted 09/22/09; revised 03/08/10; accepted 03/12/10. Author for correspondence: Min-Chie Chiu (e-mail:minchie.chiu@msa.hinet.net).

*Department of Automatic Control Engineering, ChungChou Institute of Technology, Changhua County, Taiwan, R.O.C.

**Department of Mechanical Engineering, Tatung University, Taipei, Taiwan, R.O.C. inlet tubes as well as perforated holes), the perforated ratio, and the length of perforated tube decrease. Consequently, a successful approach used for the optimal design of the twochamber mufflers equipped with perforated plug/non-plug tubes under space and back pressure constrained conditions has been demonstrated.

\section{INTRODUCTION}

In the past three decades, to increase acoustical performance, an assessment of a new acoustical element, an internal perforated tube which is an essential acoustical element used to depress low frequency sound energy, was introduced and discussed by Sullivan and Crocker in 1978 [23]. Based on the coupled equations derived by Sullivan and Crocker, a series of theories and numerical techniques in decoupling the acoustical problems have been proposed [6, 14, 21, 22, 24]. Concerning the flow effect, Munjal [12] and Peat [16] published the generalized decoupling and numerical decoupling methods. Munjal et al. [13] investigated the acoustical effect and the system's back pressure with respect to several design parameters for perforated plug and cross-flow perforated mufflers. However, the assessment of the muffler's optimal shape design within a constrained space has been neglected. Kar and Munjal [7] widen the assessment on a muffler hybridized by multiple interacting perforated ducts using a generalized analysis. Nishimura et al. [20] established a mathematical model for an elliptical muffler that was equipped with a perforated pipe within a higher-order mode in a zero speed flowing field. Finally, Lee and Selamet [10] expanded the BEM (Boundary Element Method) numerical assessment on a resonator with/without a fiber filled inner cavity. The above researches have provided various solutions for solving the complicated acoustical field problem for perforated mufflers. However, the assessment of a multi-chamber plug-muffler's optimal shape design within a constrained space as well as a pressure-drop limit was rarely tackled. In previous work [2-4], the shape optimization of multi-chamber mufflers equipped 
with perforated/non-perforated tubes within a constrained situation has been discussed. However, the effect of the system's back pressure, which may cause the decrement of the flow rate in the system, has been neglected.

The application of a single-chamber perforated plug muffler in reducing a venting noise has prevailed in modern industries. Yet, on the basis of experimental trial the muffler design was shown to be time-consuming and the acoustical performance to be insufficient. Additionally, mufflers equipped with multiple perforated plug tubes have higher back-pressure even though their acoustical performance is superior to muffler equipped with perforated non-plug tubes. In order to improve the performance of the noise control device and reduce the back-pressure, a hybrid muffler (a two-chamber muffler conjugated with a single perforated plug and a single non-plug tube) is investigated. Moreover, to overcome the drawback of a possible overload pressure drop in the mufflers, the specified allowable pressure drop in a muffler has been considered along with the process of $S A$ optimization. By using a simulated annealing $(S A)$ method, the muffler's performance is improved. Additionally, to investigate the acoustical effect with respect to $\Delta p_{\max }$, the shape optimization of mufflers at various $\Delta p_{\max }(100 \mathrm{~Pa}, 200 \mathrm{~Pa}, 300 \mathrm{~Pa}, 400 \mathrm{~Pa}, 500$ $\mathrm{Pa}$, and infinite $\mathrm{Pa}$ ) has also been discussed. In this paper, the numerical decoupling methods used in forming a four-pole system matrix are in line with the above $S A$ method. These, in turn, are responsible for developing a new muffler shape by adjusting the two-chamber muffler equipped with perforated plug/non-plug tubes within certain space constraints and backpressure constraints.

\section{MATHEMATICAL MODELS}

\section{System Matrix}

In this paper, two-chamber mufflers hybridized with perforated plug/non-plug tubes were adopted for noise elimination as shown in Fig. 1. The outline of the mufflers selected as the noise-reduction device is shown in Fig. 2. The recognition of acoustical elements is shown in Fig. 3. As indicated in Fig. 3 , four kinds of muffler components, including straight duct, perforated duct, expanded/perforated plug duct, and contracted/ perforated plug duct, are recognized and symbolized as I, II, III, and IV.

Consequently, the related acoustic pressure $p$ and acoustic particle velocity $u$ in the acoustical field are represented by seven nodes and shown in Fig. 4. As derived in the Appendices $\mathrm{A}, \mathrm{B}$, and $\mathrm{C}$, individual transfer matrixes with respect to a straight ducts [27], a perforated duct (Appendices A), an expanded/perforated plug duct (Appendices B), and a contracted/perforated plug duct (Appendices C) are described as follows:

$$
\left(\begin{array}{c}
p_{1} \\
\rho_{o} c_{o} u_{1}
\end{array}\right)=e^{-j M_{1} k\left(L_{1}+L_{A 1}\right) /\left(1-M_{1}^{2}\right)}\left[\begin{array}{cc}
T S 1_{1,1} & T S 1_{1,2} \\
T S 1_{2,1} & T S 1_{2,2}
\end{array}\right]\left(\begin{array}{c}
p_{2} \\
\rho_{o} c_{o} u_{2}
\end{array}\right)
$$

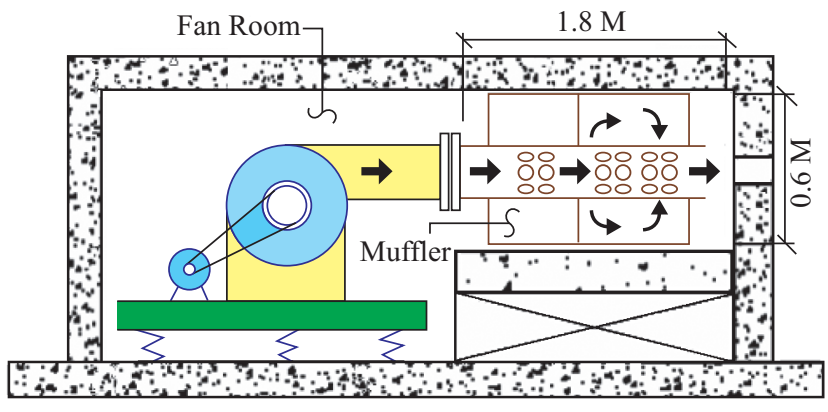

Fig. 1. The space-constrained fan room.

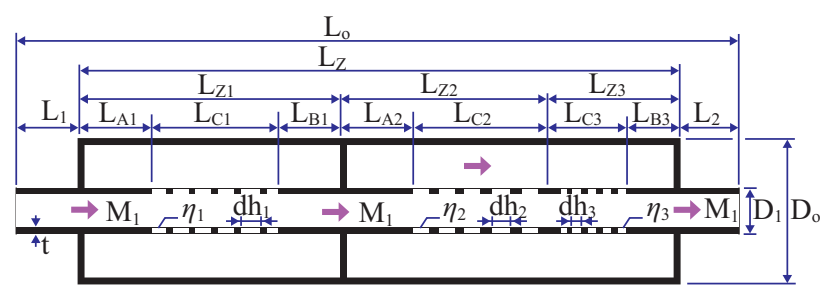

Fig. 2. The outline of a two-chamber muffler hybridized with a perforated plug/non plug tube.

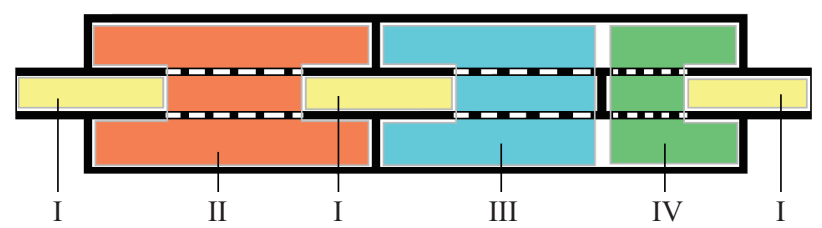

Fig. 3. The acoustical elements for a two-chamber muffler hybridized with a perforated plug/non plug tube.

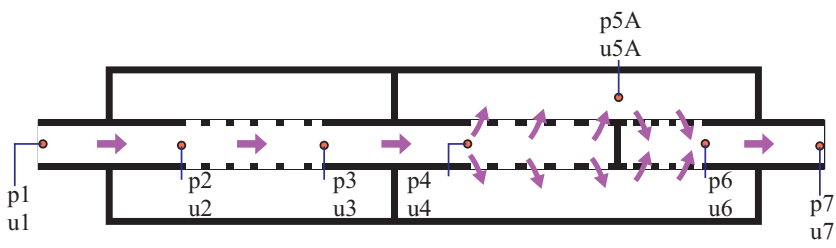

Fig. 4. The acoustical field in a two-chamber muffler hybridized with a perforated plug/non plug tube.

$$
\begin{gathered}
\left(\begin{array}{c}
p_{2} \\
\rho_{o} c_{o} u_{2}
\end{array}\right)=\left[\begin{array}{ll}
T P 2_{1,1} & T P 2_{1,2} \\
T P 2_{2,1} & T P 2_{2,2}
\end{array}\right]\left(\begin{array}{c}
p_{3} \\
\rho_{o} c_{o} u_{3}
\end{array}\right) \\
\left(\begin{array}{c}
p_{3} \\
\rho_{o} c_{o} u_{3}
\end{array}\right)=e^{-j M_{1} k\left(L_{B 1}+L_{A 2}\right) /\left(1-M_{1}^{2}\right)}\left[\begin{array}{ll}
T S 3_{1,1} & T S 3_{1,2} \\
T S 3_{2,1} & T S 3_{2,2}
\end{array}\right]\left(\begin{array}{c}
p_{4} \\
\rho_{o} c_{o} u_{4}
\end{array}\right)
\end{gathered}
$$

$$
\left(\begin{array}{c}
p_{4} \\
\rho_{o} c_{o} u_{4}
\end{array}\right)=\left[\begin{array}{cc}
T P E 4_{1,1} & T P E 4_{1,2} \\
T P E 4_{2,1} & T P E 4_{2,2}
\end{array}\right]\left(\begin{array}{c}
p_{5 A} \\
\rho_{o} c_{o} u_{5 A}
\end{array}\right)
$$




$$
\begin{gathered}
\left(\begin{array}{c}
p_{5 A} \\
\rho_{o} c_{o} u_{5 A}
\end{array}\right)=\left[\begin{array}{ll}
T P C 5_{1,1} & T P C 5_{1,2} \\
T P C 5_{2,1} & T P C 5_{2,2}
\end{array}\right]\left(\begin{array}{c}
p_{6} \\
\rho_{o} c_{o} u_{6}
\end{array}\right) \\
\left(\begin{array}{c}
p_{6} \\
\rho_{o} c_{o} u_{6}
\end{array}\right)=e^{-j M_{1} k\left(L_{B_{3}}+L_{2}\right) /\left(1-M_{1}^{2}\right)}\left[\begin{array}{ll}
T S 6_{1,1} & T S 6_{1,2} \\
T S 6_{2,1} & T S 6_{2,2}
\end{array}\right]\left(\begin{array}{c}
p_{7} \\
\rho_{o} c_{o} u_{7}
\end{array}\right)
\end{gathered}
$$

The total transfer matrix assembled by multiplication is

$$
\begin{aligned}
& \left(\begin{array}{c}
p_{1} \\
\rho_{o} c_{o} u_{1}
\end{array}\right)=e^{-j k M_{1}\left[\frac{L_{1}+L_{A 1}+L_{B 1}+L_{A 2}+L_{B 3}+L_{2}}{1-M_{1}^{2}}\right]}\left[\begin{array}{ll}
T S 1_{1,1} & T S 1_{1,2} \\
T S 1_{2,1} & T S 1_{2,2}
\end{array}\right] \\
& {\left[\begin{array}{ll}
T P 2_{1,1} & T P 2_{1,2} \\
T P 2_{2,1} & T P 2_{2,2}
\end{array}\right]\left[\begin{array}{ll}
T S 3_{1,1} & T S 3_{1,2} \\
T S 3_{2,1} & T S 3_{2,2}
\end{array}\right]\left[\begin{array}{ll}
T P E 4_{1,1} & T P E 4_{1,2} \\
T P E 4_{2,1} & T P E 4_{2,2}
\end{array}\right]} \\
& {\left[\begin{array}{ll}
T P C 5_{1,1} & T P C 5_{2,1} \\
T P C 5_{2,1} & T P C 5_{2,2}
\end{array}\right]\left[\begin{array}{ll}
T S 6_{1,1} & T S 6_{1,2} \\
T S 6_{2,1} & T S 6_{2,2}
\end{array}\right]\left(\begin{array}{c}
p_{7} \\
\rho_{o} c_{o} u_{7}
\end{array}\right)}
\end{aligned}
$$

A simplified form of a matrix is expressed as

$$
\left(\begin{array}{c}
p_{1} \\
\rho_{o} c_{o} u_{1}
\end{array}\right)=\left[\begin{array}{cc}
T_{11}^{*} & T_{12}^{*} \\
T_{21}^{*} & T_{22}^{*}
\end{array}\right]\left(\begin{array}{c}
p_{7} \\
\rho_{o} c_{o} u_{7}
\end{array}\right)
$$
[14]

The sound transmission loss (STL) of a muffler is defined as

$$
\operatorname{STL}\left(Q, f, R T_{1}, R T_{2}, R T_{3}, R T_{4}, R T_{5}, R T_{6}, R T_{7}, R T_{8}, R T_{9}, R T_{10},\right.
$$$$
\left.R T_{11}, R T_{12}, R T_{13}, \Delta p_{\max }\right)
$$$$
=20 \log \left(\frac{\left|T_{11}^{*}+T_{12}^{*}+T_{21}^{*}+T_{22}^{*}\right|}{2}\right)+10 \log \left(\frac{S_{1}}{S_{7}}\right)
$$

where

$R T_{1}=L_{Z} / L_{0} ; R T_{2}=L_{Z 1} / L_{Z} ; R T_{3}=L_{Z 2} /\left(L_{Z}-L_{Z 1}\right) ;$

$R T_{4}=L_{\mathrm{Cl}} / L_{\mathrm{Z} 1} ; R T_{5}=L_{\mathrm{C} 2} / L_{\mathrm{Z} 2} ; R T_{6}=L_{\mathrm{C} 3} / L_{\mathrm{Z} 3} ; R T_{7}=D_{1} / D_{\mathrm{o}} ;$

$R T_{8}=\eta_{1} ; R T_{9}=d h_{1} ; R T_{10}=\eta_{2} ; R T_{11}=d h_{2} ; R T_{12}=\eta_{3} ; T_{13}=d h_{3} ;$

$L_{\mathrm{A} 1}=\left(L_{\mathrm{Z} 1}-L_{\mathrm{C} 1}\right) / 2=L_{\mathrm{B} 1} ; L_{1}=L_{2}=\left(L_{\mathrm{o}}-L_{\mathrm{Z}}\right) / 2$

According to the experimental investigation of back pressure for a perforated-tube muffler and a one-chamber plug muffler by Munjal et al. [16], the individual mean pressure drops $\left(\Delta p_{1}, \Delta p_{2}\right)$ are

$$
\Delta p_{1}=\mathrm{H}_{1} *(0.87+0.06 \times 1)
$$

$$
\Delta p_{2}=\mathrm{H}_{1} *\left(5.6 \mathrm{e}^{-0.23 \times 2}+67.3 \mathrm{e}^{-3.05 \times 2}\right)
$$

To meet the system requirement of allowable maximal pressure drop $\left(\Delta p_{\max }\right)$, the total mean pressure drop $\left(\Delta p=\Delta p_{1}+\right.$ $\Delta p_{2}$ ) should be governed as

$$
\left(\Delta p_{\max }\right) \geq \Delta p
$$

\section{Overall Sound Power Level}

The overall $S W L_{\mathrm{T}}$ silenced by the muffler at the outlet is

$$
S W L_{\mathrm{T}}=10 * \log \left\{\begin{array}{ccc}
{[S W L O(f=125)-} & {[S W L O(f=250)-} \\
S T L(f=125)] / 10 & +10^{S T L(f=250)] / 10} \\
{[S W L O(f=500)-} & S W L O(f=1000)- & S W L O(f=2000)- \\
+10^{S T L(f=500)] / 10}+10^{S T L(f=1000)] / 10}+10^{S T L(f=2000)] / 10}
\end{array}\right\}
$$

where (1) $S W L O_{i}$ is the original $S W L$ at the inlet of the muffler (or pipe outlet), and $i$ is the index of the octave band frequency.

(2) $S T L_{i}$ is the muffler's $S T L$ with respect to the relative octave band frequency.

\section{Objective Function}

By using the formulas of Eqs. (9), (11) and (12), the objective function used in the $S A$ optimization with respect to each type of muffler was established.

The objective function in maximizing the $S T L$ at the pure tone $(f)$ is

$$
\begin{aligned}
O B J_{1}= & S T L\left(f, R T_{1}, R T_{2}, R T_{3}, R T_{4}, R T_{5}, R T_{6}, R T_{7}, R T_{8}, R T_{9},\right. \\
& \left.R T_{10}, R T_{11}, R T_{12}, R T_{13}, \Delta p_{\max }\right)
\end{aligned}
$$

The objective function in eliminating overall $S W L_{\mathrm{T}}$ is

$$
O B J_{2}=S W L_{\mathrm{T}}\left(R T_{1}, R T_{2}, R T_{3}, R T_{4}, R T_{5}, R T_{6}, R T_{7}, R T_{8}, R T_{9},\right.
$$

$$
\left.R T_{10}, R T_{11}, R T_{12}, R T_{13}, \Delta p_{\max }\right)
$$

\section{MODEL CHECK}

Before performing the $S A$ optimal simulation on mufflers, an accuracy check of the mathematical model on the acoustical elements of a one-chamber perforated muffler and a onechamber perforated plug muffler are performed using the experimental data from Sullivan et al. and Munjal [14, 21-23]. As depicted in Figs. 5 and 6, accuracies between the theoretical and experiment data are in agreement. Therefore, the proposed fundamental mathematical models are acceptable. Consequently, the model linked with the numerical method is applied to the shape optimization in the following section. Therefore, the proposed fundamental mathematical model is applicable. 


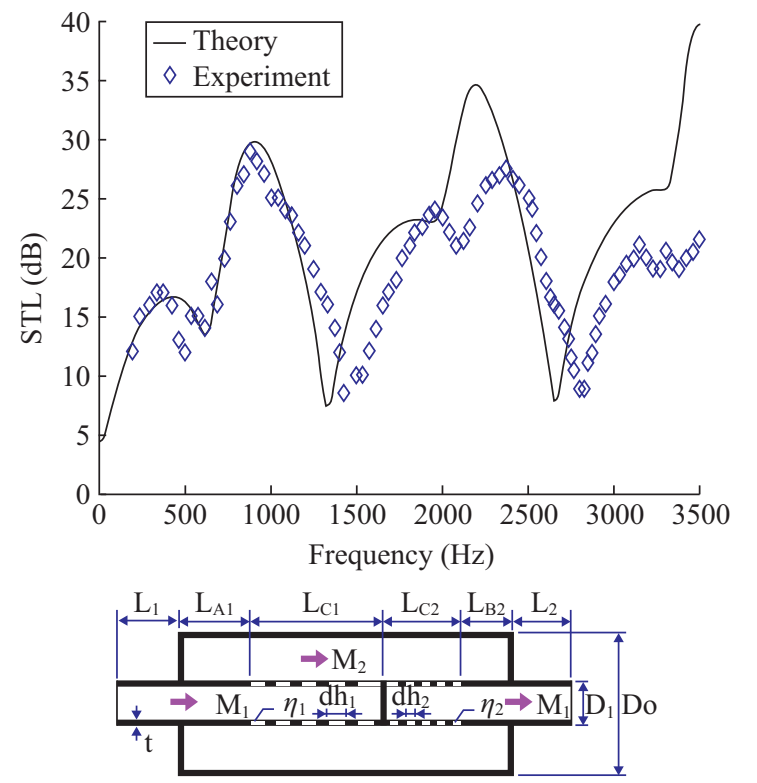

Fig. 5. Performance of a one-chamber perforated plug muffler with the mean flow $\left[M_{1}=M_{2}=0.05, D_{1}=0.0493(\mathrm{~m}), D 0=0.1016(\mathrm{~m}), L_{C 1}=\right.$ $L_{C 2}=0.1286(\mathrm{~m}), L_{1}=L_{2}=0.1(\mathrm{~m}), L_{\mathrm{A} 1}=L_{\mathrm{B} 2}=0.0(\mathrm{~m}), t=0.0081$ $\left.(\mathrm{m}), d h_{1}=d h_{2}=0.00249(\mathrm{~m}), \eta_{1}=\eta_{2}=0.037\right][$ Experimental data is from Sullivan and Munjal [14, 21, 22]].
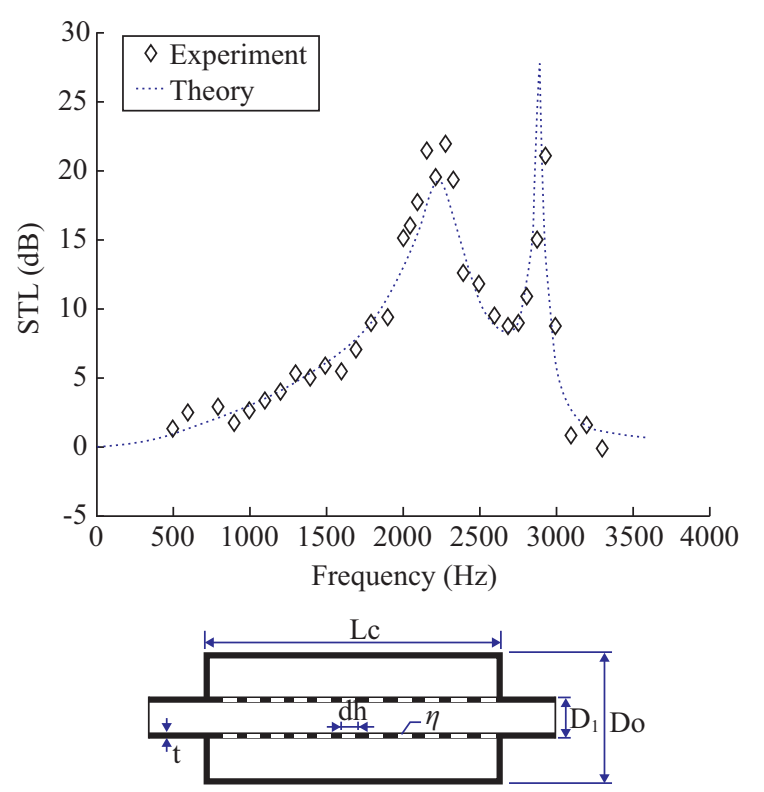

Fig. 6. Performance of a single-chamber perforated muffler without the mean flow $\left[D_{1}=0.058(\mathrm{~m}), D o=0.0762(\mathrm{~m}), L_{c}=0.0667(\mathrm{~m}), \mathrm{t}=\right.$ $0.0081(\mathrm{~m}), \mathrm{dh}=0.00249(\mathrm{~m}), \eta=0.037]$ [Experiment data is from Sullivan and Crocker [23]].

\section{CASE STUDY}

In this paper, a muffler confined inside a fan room is shown in Fig. 1. The octave band of the fan's sound power level inside the pipe outlet (muffler's inlet) is listed in Table 1. To efficiently depress the sound energy, a muffler with a onechamber perforated tube as well as a one-chamber perforated
Table 1. The spectrum of an exhausted sound power level $(S W L)$.

\begin{tabular}{|ccccccc|}
\hline$f(\mathrm{~Hz})$ & 125 & 250 & 500 & $1 \mathrm{k}$ & $2 \mathrm{k}$ & Overall \\
(a) $\mathbf{S W L O - d B}$ & 110 & 125 & 120 & 105 & 100 & 126.3 \\
\hline
\end{tabular}

Table 2. Range of design parameters for a two-chamber muffler with perforated plug/non-plug tubes.

\begin{tabular}{|l|l|}
\hline & \multicolumn{1}{|c|}{ Range of design parameters } \\
\hline A two-chamber & Targeted $f=500(\mathrm{~Hz}) ; Q=0.01\left(\mathrm{~m}^{3} / \mathrm{s}\right) ; L_{\mathrm{o}}=1.8(\mathrm{~m}) ;$ \\
muffler with & $D_{\mathrm{o}}=0.6(\mathrm{~m}) ; R T_{1}:[0.2,0.8] ; R T_{2}:[0.3,0.7] ;$ \\
perforated & $R T_{3}:[0.3,0.7] ; R T_{4}:[0.2,0.8] ; R T_{5}:[0.2,0.8] ;$ \\
plug/non-plug & $R T_{6}:[0.2,0.8] ; R T_{7}:[0.2,0.8] ; R T_{8}:[0.03,0.1] ;$ \\
tubes & $R T_{9}:[0.00175,0.007] ; R T_{10}:[0.03,0.1] ;$ \\
& $R T_{11}:[0.00175,0.007] ; R T_{12}:[0.03,0.1] ;$ \\
& $R T_{13}:[0.00175,0.007] ; \Delta p_{\max }: 100 \sim 500(\mathrm{~Pa})$ \\
\hline
\end{tabular}

plug tube is adopted. As shown in Fig. 1, the available space for a muffler is $0.6 \mathrm{~m}$ in width, $0.6 \mathrm{~m}$ in height, and $1.8 \mathrm{~m}$ in length. The allowable pressure drop for the muffler is between 100-500 Pa. To simplify the optimization, the flow rate $(Q=$ $\left.0.01\left(\mathrm{~m}^{3} / \mathrm{s}\right)\right)$ and thickness of the perforated tube $(t=0.0015$ (m)) are preset in advance. The corresponding space constraints and the ranges of design parameters are summarized in Table 2. Before the minimization of the broadband noise is performed, the maximization of the STL at the targeted pure tone $(500 \mathrm{~Hz})$ has been performed for the purpose of an accuracy check on the $S A$ method.

\section{OPTIMIZATION}

Various methods used for solving optimization problems can be classified into three categories - enumerative, deterministic and stochastic. The first techniques are best applied to problems that are defined with a few discrete decision variables only $[9,18]$. The second techniques mainly incorporate problem domain knowledge to reduce the size of the search space. However, during the optimization process $[19,25,26]$, the gradient methods, one of the deterministic techniques, requires a starting point or a mathematical derivation that is calculated in advance. Evolutionary Algorithms (EAs) belong to the group of stochastic search methods, also referred to as random search. Evolutionary Algorithms have been widely developed for two decades. Many good EAs have been established. Simulated Annealing [5] is one of the best stochastic search methods. Here, sensitivity analyses is not necessary for choosing the starting design data, which is required in classical gradient methods of EPFM, IPFM and FDM [1]. Therefore, $S A$ is adopted as an optimizer and used in the muffler's shape optimization.

The basic concept behind $S A$ was first introduced by Metropolis et al. [11] and developed by Kirkpatrick et al. [8]. As indicated in Fig. 7, for a simulated annealing $(S A)$ method, 


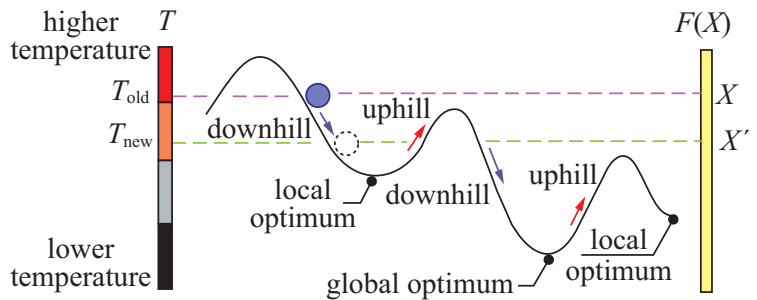

Fig. 7. $S A$ algorithm from a physical viewpoint.

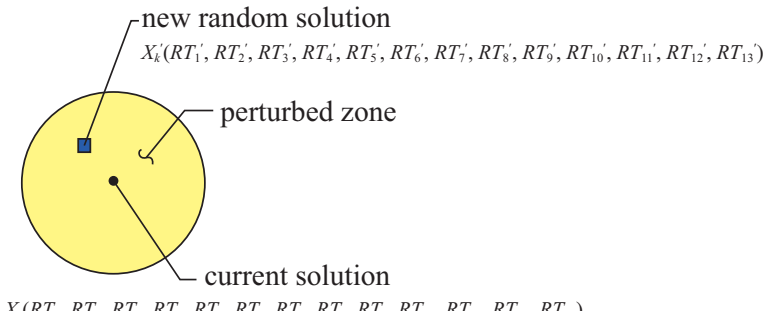

Fig. 8. New random solution in a perturbed zone.

each point $X$ of the search space is compared to a state of some physical system, and the function $F(X)$ to be minimized is interpreted as the internal energy of the system in that state. Therefore, the goal is to bring the system from an arbitrary initial state to a state with the minimum possible energy. Annealing is the process of heating and keeping a metal at a stabilized temperature while cooling it slowly. Slow cooling allows the particles to keep their state close to the minimal energy state. The algorithm starts by generating a random initial solution. The scheme of $S A$ is a variation of the hillclimbing algorithm. All downhill movements for improvement are accepted for the decrement of the system's energy. In order to escape from the local optimum, $S A$ also allows movement resulting in solutions that are worse (uphill moves) than the current solution. To imitate the evolution of the $S A$ algorithm, a new random solution $\left(X^{\prime}\right)$ shown in Fig. 8 is chosen from the neighborhood of the current solution $(X)$.

If the change in the objective function (or energy) is negative $(\Delta F \leq 0)$, the new solution will be acknowledged as the new current solution with the transition property $\left(p b\left(X^{\prime}\right)\right)$ of 1 . If the change is not negative $(\Delta F>0)$, the probability of making the transition to the new state $X^{\prime}$ will be a function $p b(\Delta F / C T)$ of the energy difference $\Delta F=F\left(X^{\prime}\right)-F(X)$ between the two states and a function of the global time-varying parameter $T$. The new transition property $\left(p b\left(X^{\prime}\right)\right)$ varies from $0 \sim 1$ will be calculated by the Boltzmann's factor $\left(p b\left(X^{\prime}\right)=\right.$ $\exp (\Delta F / C T))$ as shown in Eq. (15)

$$
\begin{gathered}
p b\left(X^{\prime}\right)=\left(\begin{array}{c}
1, \Delta F \leq 0 \\
\exp \left(\frac{-\Delta F}{C T}\right), \Delta f>0 \\
\Delta F=F\left(X^{\prime}\right)-F(X)
\end{array}\right.
\end{gathered}
$$

Table 3. The pseudo-code implementing the simulated annealing heuristic.
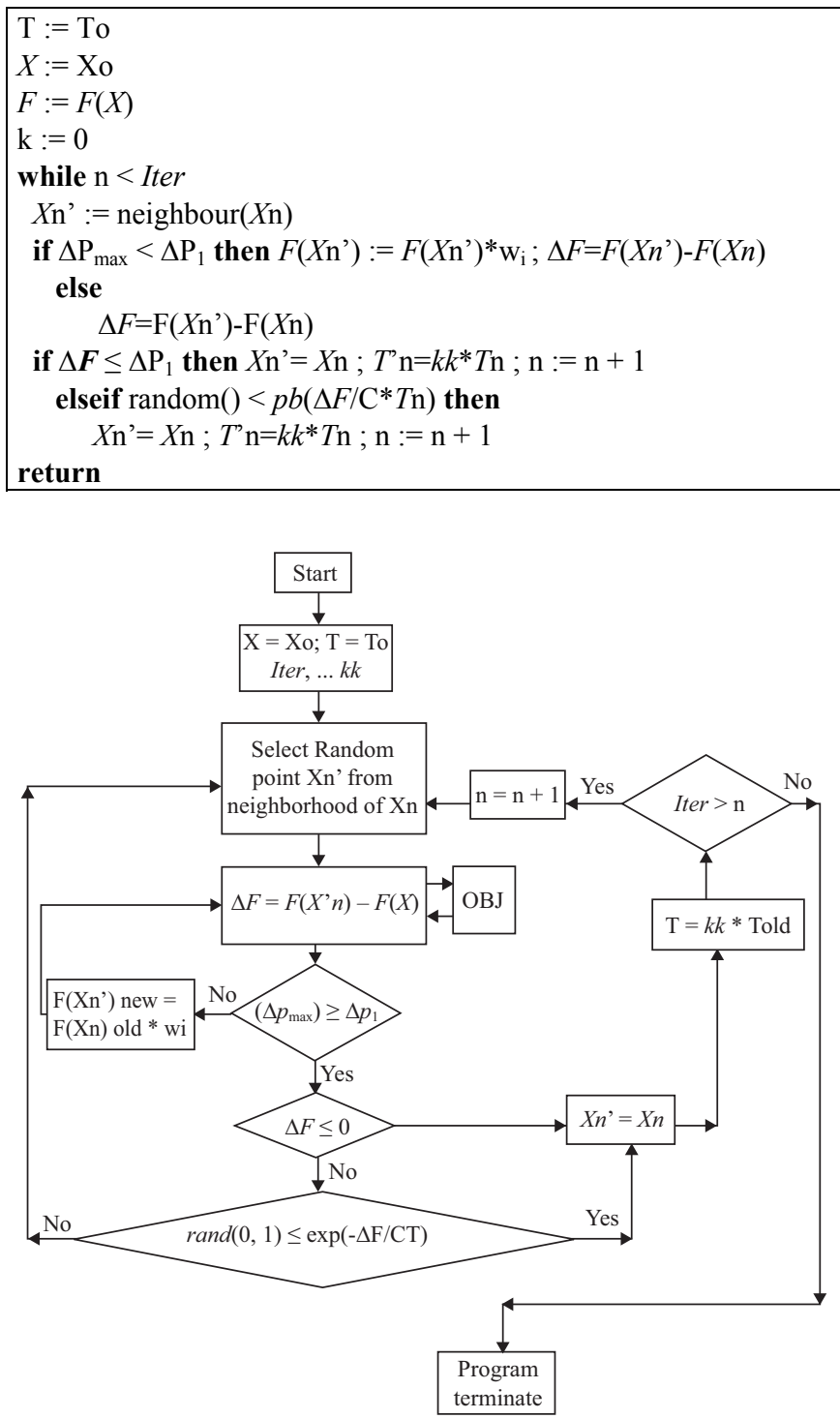

Fig. 9. Flow diagram of a $S A$ optimization.

where $C$ and $T$ are the Boltzmann constant and the current temperature. Moreover, compared with the new random probability of rand $(0,1)$, if the transition property $\left(p b\left(X^{\prime}\right)\right)$ is greater than a random number of rand $(0,1)$, the new solution (worse solution) which results in a higher energy condition will then be accepted; otherwise, it is rejected. Each successful substitution of the new current solution leads to the decay of the current temperature as

$$
T_{\text {new }}=k k^{*} T_{\text {old }}
$$

where $k k$ is the cooling rate.

The flow diagram of the $S A$ optimization is described and shown in Fig. 9. In addition, the pseudo-code implementing the simulated annealing heuristic is listed in Table 3 . The 
Table 4. Optimal design data for a muffler with perforated plug/ non-plug tubes (targeted tone at $500 \mathrm{~Hz}$ ).

\begin{tabular}{|c|c|c|c|c|c|c|c|c|c|c|}
\hline \multicolumn{2}{|c|}{$S A$ parameter } & \multirow{2}{*}{\multicolumn{7}{|c|}{ Design parameters }} & \multicolumn{2}{|c|}{ Performance } \\
\hline Iter & $k k$ & & & & & & & & \multirow[t]{2}{*}{$S T L(\mathrm{~dB})$} & \multirow[t]{2}{*}{$\Delta p_{\max }(\mathrm{Pa})$} \\
\hline \multirow{4}{*}{50} & \multirow{4}{*}{0.91} & $R T_{1}$ & $R T_{2}$ & $R T_{3}$ & $R T_{4}$ & $R T_{5}$ & $R T_{6}$ & $R T_{7}$ & & \\
\hline & & 0.7262 & 0.6508 & 0.6508 & 0.7262 & 0.7262 & 0.7262 & 0.7262 & \multirow{3}{*}{34.4} & \multirow{3}{*}{2.4} \\
\hline & & $R T_{8}$ & $R T_{9}$ & $R T_{10}$ & $R T_{11}$ & $R T_{12}$ & $R T_{13}$ & & & \\
\hline & & 0.09138 & 0.0063 & 0.09138 & 0.00635 & 0.0913 & 0.006354 & & & \\
\hline \multirow{4}{*}{50} & \multirow{4}{*}{0.93} & $R T_{1}$ & $R T_{2}$ & $R T_{3}$ & $R T_{4}$ & $R T_{5}$ & $R T_{6}$ & $R T_{7}$ & \multirow{4}{*}{61.4} & \multirow{4}{*}{54.7} \\
\hline & & 0.3653 & 0.4102 & 0.4102 & 0.3653 & 0.3653 & 0.3653 & 0.3653 & & \\
\hline & & $R T_{8}$ & $R T_{9}$ & $R T_{10}$ & $R T_{11}$ & $R T_{12}$ & $R T_{13}$ & & & \\
\hline & & 0.09138 & 0.0063 & 0.09138 & 0.00635 & 0.0913 & 0.006354 & & & \\
\hline \multirow{4}{*}{50} & \multirow{4}{*}{0.95} & $R T_{1}$ & $R T_{2}$ & $R T_{3}$ & $R T_{4}$ & $R T_{5}$ & $R T_{6}$ & $R T_{7}$ & \multirow{4}{*}{73.3} & \multirow{4}{*}{191.6} \\
\hline & & 0.2752 & 0.3501 & 0.3501 & 0.2752 & 0.2752 & 0.2752 & 0.2752 & & \\
\hline & & $R T_{8}$ & $R T_{9}$ & $R T_{10}$ & $R T_{11}$ & $R T_{12}$ & $R T_{13}$ & & & \\
\hline & & 0.03878 & 0.0024 & 0.03878 & 0.00240 & 0.0387 & 0.002408 & & & \\
\hline \multirow{4}{*}{50} & \multirow{4}{*}{0.97} & $R T_{1}$ & $R T_{2}$ & $R T_{3}$ & $R T_{4}$ & $R T_{5}$ & $R T_{6}$ & $R T_{7}$ & \multirow{4}{*}{90.8} & \multirow{4}{*}{12.8} \\
\hline & & 0.5011 & 0.5007 & 0.5007 & 0.5011 & 0.5011 & 0.5011 & 0.5011 & & \\
\hline & & $R T_{8}$ & $R T_{9}$ & $R T_{10}$ & $R T_{11}$ & $R T_{12}$ & $R T_{13}$ & & & \\
\hline & & 0.06513 & 0.0043 & 0.06513 & 0.00438 & 0.0651 & 0.004385 & & & \\
\hline \multirow{4}{*}{50} & \multirow{4}{*}{0.99} & $R T_{1}$ & $R T_{2}$ & $R T_{3}$ & $R T_{4}$ & $R T_{5}$ & $R T_{6}$ & $R T_{7}$ & \multirow{4}{*}{87.2} & \multirow{4}{*}{15.5} \\
\hline & & 0.4811 & 0.4874 & 0.4874 & 0.4811 & 0.4811 & 0.4811 & 0.4811 & & \\
\hline & & $R T_{8}$ & $R T_{9}$ & $R T_{10}$ & $R T_{11}$ & $R T_{12}$ & $R T_{13}$ & & & \\
\hline & & 0.06279 & 0.0042 & 0.06279 & 0.00420 & 0.0627 & 0.004209 & & & \\
\hline \multirow{4}{*}{200} & & $R T_{1}$ & $R T_{2}$ & $R T_{3}$ & $R T_{4}$ & $R T_{5}$ & $R T_{6}$ & $R T_{7}$ & & \\
\hline & 007 & 0.5381 & 0.5254 & 0.5254 & 0.5381 & 0.5381 & 0.5381 & 0.5381 & 071 & 02 \\
\hline & $\underline{0.91}$ & $R T_{8}$ & $R T_{9}$ & $R T_{10}$ & $R T_{11}$ & $R T_{12}$ & $R T_{13}$ & & 91.1 & 9.3 \\
\hline & & 0.06944 & 0.0047 & 0.06944 & 0.00470 & 0.0694 & 0.004708 & & & \\
\hline & & $R T_{1}$ & $R T_{2}$ & $R T_{3}$ & $R T_{4}$ & $R T_{5}$ & $R T_{6}$ & $R T_{7}$ & & \\
\hline & & 0.2406 & 0.3271 & 0.3271 & 0.2406 & 0.2406 & 0.2406 & 0.2406 & & \\
\hline 400 & $\underline{0.91}$ & $R T_{8}$ & $R T_{9}$ & $R T_{10}$ & $R T_{11}$ & $R T_{12}$ & $R T_{13}$ & & 104 & 342.5 \\
\hline & & 0.03474 & 0.0021 & 0.03474 & 0.00210 & 0.0347 & 0.002106 & & & \\
\hline & & $R T_{1}$ & $R T_{2}$ & $R T_{3}$ & $R T_{4}$ & $R T_{5}$ & $R T_{6}$ & $R T_{7}$ & & \\
\hline & & $\underline{0.2303}$ & $\underline{0.3202}$ & $\underline{0.3202}$ & $\underline{0.2303}$ & $\underline{0.2303}$ & $\underline{0.2303}$ & $\underline{0.2303}$ & & 111 \\
\hline$\underline{800}$ & $\underline{0.97}$ & $R T_{8}$ & $R T_{9}$ & $R T_{10}$ & $R T_{11}$ & $R T_{12}$ & $R T_{13}$ & & 133 & 413.1 \\
\hline & & $\underline{0.2303}$ & $\underline{0.0335}$ & $\underline{0.00201}$ & $\underline{0.03353}$ & $\underline{0.0020}$ & $\underline{0.03353}$ & & & \\
\hline Note & $T_{1}=$ & $T_{2}-$ & ; $R T_{3}=$ & $\begin{array}{l}\left.L_{\mathrm{Z}}-L_{\mathrm{Z} 1}\right) \\
=d h_{3} .\end{array}$ & $=L_{\mathrm{Cl}} / L_{\mathrm{Z}}$ & $=L_{\mathrm{C} 2} / L$ & $\Gamma_{6}=L_{\mathrm{C} 3} / L$ & $7=D_{1}$ & $R T_{8}=\eta$ & $R T_{9}=d h_{1}$ \\
\hline
\end{tabular}

process is repeated until the pre-determined number (Iter) of the outer loop is reached. Obviously, the effect of the state energies on the system's evolution depends on the temperature. The evolution is sensitive only to coarser energy variations when $T$ is large and to finer variations when $T$ is small.

During the optimization process, the back pressure $(\Delta p)$ will be also calculated and compared with a limit of $\Delta p_{\max }$. If
$\Delta p$ is smaller than $\Delta p_{\max }$, the current solution will be valid and used for further iteration. If this is not the case, the value of the objective function will be weighted by $w_{\mathrm{i}}$ to discard the current solution. The algorithm repeats the perturbation of the current solution and the measurement of the change in the OBJ. To reach an initial transition probability of 0.5 , the initial temperature $(T o)$ is selected as $0.2[15]$. 
Table 5. Optimal design data for a muffler with perforated plug/non-plug tubes at various maximal back pressures (broadband noise) $($ Iter $=800 ; k k=0.97)$.

\begin{tabular}{|c|c|c|c|c|c|c|c|c|c|}
\hline Cases & \multirow{2}{*}{\multicolumn{7}{|c|}{ Design parameters }} & \multicolumn{2}{|c|}{ Performance } \\
\hline Max. Back & & & & & & & & $S W L_{\mathrm{T}}$ & $\Delta p_{\max }$ \\
\hline \multirow{4}{*}{100} & $R T_{1}$ & $R T_{2}$ & $R T_{3}$ & $R T_{4}$ & $R T_{5}$ & $R T_{6}$ & $R T_{7}$ & \multirow{4}{*}{68.4} & \multirow{4}{*}{98.6} \\
\hline & 0.3202 & 0.3801 & 0.3801 & 0.3202 & 0.3202 & 0.3202 & 0.3202 & & \\
\hline & $R T_{8}$ & $R T_{9}$ & $R T_{10}$ & $R T_{11}$ & $R T_{12}$ & $R T_{13}$ & & & \\
\hline & 0.04402 & 0.0028 & 0.04402 & 0.002801 & 0.0440 & 0.002801 & & & \\
\hline \multirow{4}{*}{200} & $R T_{1}$ & $R T_{2}$ & $R T_{3}$ & $R T_{4}$ & $R T_{5}$ & $R T_{6}$ & $R T_{7}$ & \multirow{4}{*}{52.7} & \multirow{4}{*}{199.4} \\
\hline & 0.2727 & 0.3485 & 0.3485 & 0.2727 & 0.2727 & 0.2727 & 0.2727 & & \\
\hline & $R T_{8}$ & $R T_{9}$ & $R T_{10}$ & $R T_{11}$ & $R T_{12}$ & $R T_{13}$ & & & \\
\hline & 0.03849 & 0.0023 & 0.03849 & 0.002386 & 0.0384 & 0.002386 & & & \\
\hline \multirow{4}{*}{300} & $R T_{1}$ & $R T_{2}$ & $R T_{3}$ & $R T_{4}$ & $R T_{5}$ & $R T_{6}$ & $R T_{7}$ & \multirow{4}{*}{45.4} & \multirow{4}{*}{280.6} \\
\hline & 0.2520 & 0.3347 & 0.3347 & 0.2525 & 0.2525 & 0.2525 & 0.2525 & & \\
\hline & $R T_{8}$ & $R T_{9}$ & $R T_{10}$ & $R T_{11}$ & $R T_{12}$ & $R T_{13}$ & & & \\
\hline & 0.03607 & 0.0022 & 0.03607 & 0.002205 & 0.0360 & 0.002205 & & & \\
\hline \multirow{4}{*}{400} & $R T_{1}$ & $R T_{2}$ & $R T_{3}$ & $R T_{4}$ & $R T_{5}$ & $R T_{6}$ & $R T_{7}$ & \multirow{4}{*}{40.4} & \multirow{4}{*}{365.4} \\
\hline & 0.2370 & 0.3246 & 0.3246 & 0.2370 & 0.2370 & 0.2370 & 0.2370 & & \\
\hline & $R T_{8}$ & $R T_{9}$ & $R T_{10}$ & $R T_{11}$ & $R T_{12}$ & $R T_{13}$ & & & \\
\hline & 0.03431 & 0.0020 & 0.03431 & 0.002073 & 0.0343 & 0.002073 & & & \\
\hline \multirow{4}{*}{500} & $R T_{1}$ & $R T_{2}$ & $R T_{3}$ & $R T_{4}$ & $R T_{5}$ & $R T_{6}$ & $R T_{7}$ & \multirow{4}{*}{36.0} & \multirow{4}{*}{460.6} \\
\hline & 0.2245 & 0.3163 & 0.3163 & 0.2245 & 0.2245 & 0.2245 & 0.2245 & & \\
\hline & $R T_{8}$ & $R T_{9}$ & $R T_{10}$ & $R T_{11}$ & $R T_{12}$ & $R T_{13}$ & & & \\
\hline & 0.03286 & 0.0019 & 0.03286 & 0.001964 & 0.0328 & 0.001964 & & & \\
\hline \multirow{4}{*}{ - } & $R T_{1}$ & $R T_{2}$ & $R T_{3}$ & $R T_{4}$ & $R T_{5}$ & $R T_{6}$ & $R T_{7}$ & \multirow{4}{*}{33.2} & \multirow{4}{*}{534.3} \\
\hline & 0.2168 & 0.3112 & 0.3112 & 0.2168 & 0.2168 & 0.2168 & 0.2168 & & \\
\hline & $R T_{8}$ & $R T_{9}$ & $R T_{10}$ & $R T_{11}$ & $R T_{12}$ & $R T_{13}$ & & & \\
\hline & 0.03196 & 0.0018 & 0.03196 & 0.001897 & 0.0319 & 0.001897 & & & \\
\hline \multicolumn{10}{|c|}{$\begin{aligned} \text { Notes: } & R T_{1}=L_{\mathrm{Z}} / L_{\mathrm{o}} ; R T_{2}=L_{\mathrm{Z} 1} / L_{\mathrm{Z}} ; R T_{3}=L_{\mathrm{Z} 2} /\left(L_{\mathrm{Z}}-L_{\mathrm{Z} 1}\right) ; R T_{4}=L_{\mathrm{C} 1} / L_{\mathrm{Z} 1} ; R T_{5}=L_{\mathrm{C} 2} / L_{\mathrm{Z} 2} ; R T_{6}=L_{\mathrm{C} 3} / L_{\mathrm{Z} 3} ; R T_{7}=D_{1} / D_{\mathrm{o}} ; R T_{8}=\eta_{1} ; R T_{9}=d h_{1} ; R T_{10}= \\
& \eta_{2} ; R T_{11}=d h_{2} ; R T_{12}=\eta_{3} ; R T_{13}=d h_{3}\end{aligned}$} \\
\hline
\end{tabular}

\section{RESULTS AND DISCUSSION}

\section{Results}

The accuracy of the $S A$ optimization depends on the cooling rate $(k k)$ and the number of iterations (Iter). To achieve good optimization, both the cooling rate $(k k)$ and the number of iterations (Iter) are varied step by step

$k k=(0.91,0.93,0.95,0.97,0.99) ;$ Iter $=(50,200,400,800)$

The results of two kinds of optimizations (one, a pure tone noise; the other, a broadband noise) are described as follows:

\section{1) Pure Tone Noise Optimization}

By using Eq. (13), the maximization of the $S T L$ with respect to a two-chamber muffler hybridized with plug/non-plug tubes at the specified pure tone $(500 \mathrm{~Hz})$ was performed first.
As indicated in Table 4, eight sets of $S A$ parameters are tried in the muffler's optimization. Obviously, the optimal design data can be obtained from the last set of $S A$ parameters at $(k k$, Iter $)=(0.97,800)$. Using the optimal design data in a theoretical calculation, the resultant curves of the $S T L$ with respect to various $S A$ parameters $(k k$, Iter) is depicted in Fig. 10. As revealed in Fig. 10, the $S T L$ is precisely maximized at the desired frequency.

\section{2) Broadband Noise Optimization}

By using the formulas of Eqs. (14) and the $S A$ parameters of $(k k=0.97$, Iter $=800)$, the optimization process for minimizeing the sound power level at the muffler's outlet under limited back pressure is performed. Six kinds of $\Delta p_{\max }$ situations (100 $\mathrm{Pa}, 200 \mathrm{~Pa}, 300 \mathrm{~Pa}, 400 \mathrm{~Pa}, 500 \mathrm{~Pa}$, and infinite $\mathrm{Pa}$ ) have been considered. The optimal result at various $\Delta p_{\max }$ is obtained and summarized in Table 5. Using these optimal design data 


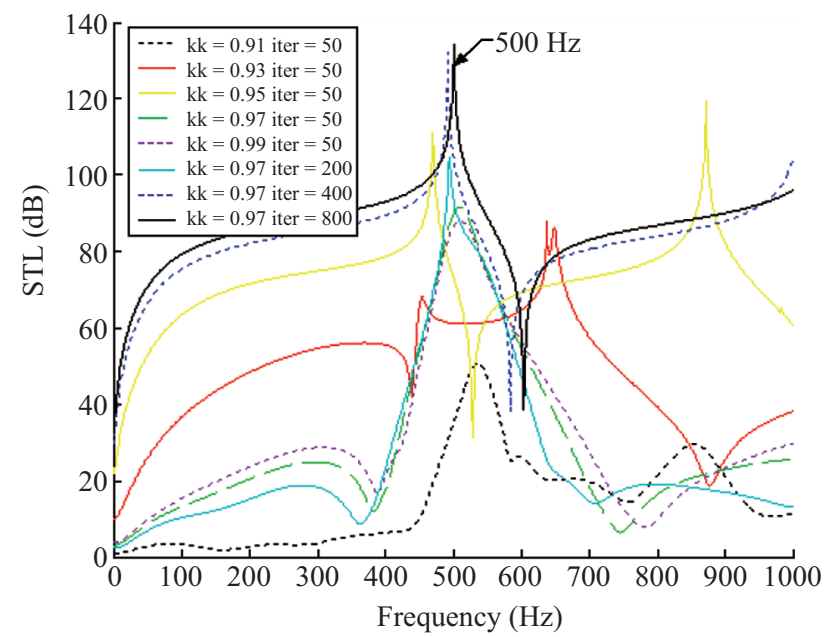

Fig. 10. The $S T L$ with respect to frequencies at various SA parameters (targeted tone: $\mathbf{5 0 0 ~} \mathbf{H z}$ ).

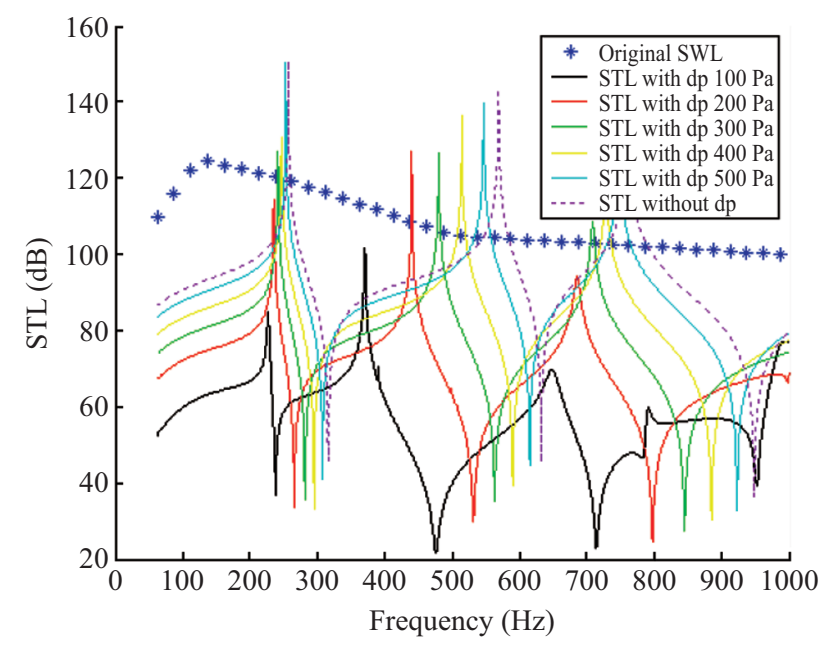

Fig. 11. Optimal $S T L$ for mufflers designed at various maximal back pressures (broadband noise).

in a theoretical calculation, the resultant curves of the $S W L$ with respect to various $\Delta p_{\max }$ are plotted in Fig. 11. As illustrated in Table 5 , the acoustical performance will be improved when the allowable system back pressure $\left(\Delta p_{\max }\right)$ is increased. Ignoring the back pressure constraint, i.e., $\Delta p_{\max }$ is infinite, the resultant silenced $S W L$ will reach the lowest value of $33.6 \mathrm{~dB}$. However, its pressure drop reaches 534.3 $\mathrm{Pa}$ which exceeds the allowable maximal back pressure of $500 \mathrm{~Pa}$ by $34.3 \mathrm{~Pa}$. To meet the back pressure criteria, a fifth set $\left(\Delta p_{\max }=500 \mathrm{~Pa}\right)$ in which the resultant pressure drop and silenced $S W L$ reach 460.6 $\mathrm{Pa}$ and $36.0 \mathrm{~dB}$ is selected.

\section{Discussion}

To achieve a sufficient optimization, the selection of the appropriate $S A$ parameter set is essential. As indicated in Table 4 and Fig. 10, the best $S A$ set with respect to a two-chamber muffler hybridized with perforated plug/non-plug tubes at the targeted pure tone noise of $500 \mathrm{~Hz}$ has been shown. Fig. 10 reveals the predicted maximal value of the $S T L$ is precisely located at the desired frequency. Therefore, the usage of the $S A$ optimization in finding a better design solution is reliable; moreover, all the pressure drops calculated in mufflers can meet the maximal allowable back pressure of $500(\mathrm{~Pa})$. Additionally, in dealing with the broadband noise using twochamber mufflers hybridized with perforated plug/non-plug tubes, the investigations into the influence of acoustical performance with respect to the system's back pressure $\left(\Delta p_{\max }=\right.$ $100 \mathrm{~Pa}, 200 \mathrm{~Pa}, 300 \mathrm{~Pa}, 400 \mathrm{~Pa}, 500 \mathrm{~Pa}$, and infinite $\mathrm{Pa}$ ) are shown in Table 5 and Fig. 11. As indicated in Table 4, the pressure drop and the acoustical performance will increase simultaneously when the diameters (at the inner tubes and the perforated holes), the perforated ratio, and the length of perforated tube decrease. In addition, the acoustical performance of the muffler will also increase when the $\Delta p_{\max }$ allowed in the venting system is enlarged. As shown in Fig. 11, the acoustical performance will increase when the allowable system back pressure $\left(\Delta p_{\max }\right)$ increases.

\section{CONCLUSION}

It has been shown that the two-chamber plug/non-plug mufflers in conjunction with a $S A$ optimizer can be easily and efficiently optimized under space and $\Delta p_{\max }$ limits by using a generalized decoupling technique, plane wave theory, as well as a four-pole transfer matrix. As indicated in Table 4, eight kinds of $S A$ parameters $(k k$, Iter) play essential roles in the solution's accuracy during $S A$ optimization. As indicated in Fig. 10, the $S T L$ is precisely maximized at the desired frequency; therefore, the tuning ability established by adjusting design parameters of the mufflers is reliable. In addition, the appropriate acoustical performance curve of the mufflers with respect to the system's back pressure $\left(\Delta p_{\max }=100 \mathrm{~Pa}, 200 \mathrm{~Pa}\right.$, $300 \mathrm{~Pa}, 400 \mathrm{~Pa}, 500 \mathrm{~Pa}$, and infinite $\mathrm{Pa}$ ) in decreasing overall broadband noise has been assessed and shown in Table 5 and Fig. 11. Results reveal that the pressure drop and the acoustical performance will increase simultaneously when the diameters (at the inner tubes and the perforated holes), the perforated ratio, and the length of perforated tube decrease. Moreover, the acoustical performance of the muffler will also increase when the $\Delta p_{\max }$ allowed in the venting system is enlarged. Therefore, to efficiently promote the acoustical performance of the two-chamber plug/non-plug muffler, the increment of $\Delta p_{\max }$ and the decrement of diameter (tubes and holes), the perforated ratio, and the length of perforated tube is necessary. Consequently, to meet the requirement of the system's $\Delta p_{\max }$, a compromise between the $S T L$ and $\Delta p$ is compulsory during $S A$ optimization. This approach used for the optimal design of the $S T L$ proposed in this study is easy and quite effective.

\section{ACKNOWLEDGMENTS}

The authors acknowledge the financial support of the National Science Council (NSC97-2221-E-235-001), ROC. 


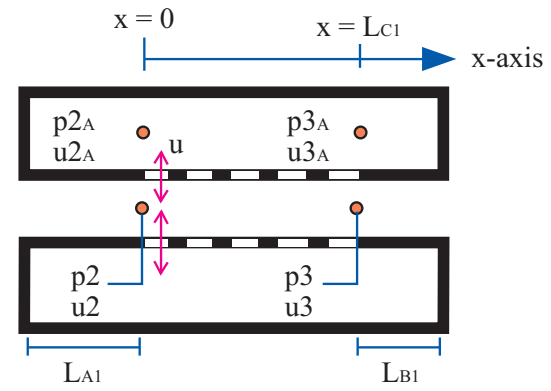

Fig. 12. Mechanism of a perforated tube.

\section{APPENDIX A - Transfer Matrix of Perforated Duct}

As indicated in Fig. 12, the perforated resonator is composed of an inner perforated tube and an outer resonating chamber. Based on Sullivan and Crocker's derivation [23], the continuity equations and momentum equations with respect to inner and outer tubes in a concentric resonator are shown below.

\section{Inner tube:}

continuity equation

$$
V \frac{\partial \rho_{2}}{\partial x}+\rho_{o} \frac{\partial u_{2}}{\partial x}+\frac{4 \rho_{o}}{D_{1}} u+\frac{\partial \rho_{2 A}}{\partial t}=0
$$

momentum equation

$$
\rho_{o}\left(\frac{\partial}{\partial t}+V \frac{\partial}{\partial x}\right) u_{2}+\frac{\partial p_{2}}{\partial x}=0
$$

\section{Outer tube:}

continuity equation

$$
\rho_{o} \frac{\partial u_{2 A}}{\partial x}-\frac{4 D_{1} \rho_{o}}{D_{o}^{2}-D_{1}^{2}} u+\frac{\partial \rho_{2 A}}{\partial t}=0
$$

momentum equation

$$
\rho_{o} \frac{\partial u_{2 A}}{\partial t}+\frac{\partial \rho_{2 A}}{\partial x}=0
$$

Assuming that the perforation along the inner tube is uniform $\left(d \xi_{1} / d x=0\right)$, the acoustic impedance of the perforation $\left(\rho_{o} c_{o} \xi_{1}\right)$ is

$$
\rho_{o} c_{o} \xi_{1}=\frac{p_{2}(x)-p_{2 A}(x)}{u(x)}
$$

where $\xi_{1}$ is the specific acoustical impedance of the perforated tube. According to the formula $\xi_{1}$ developed by Sullivan and Rao [17], the empirical formulations for the perforates, with or without mean flow, are adopted in this study.
By substituting Eq. (A5) into Eqs. (A1) (A4), eliminating $u_{2}$ and $u_{2 A}$, and expressing in a matrix form, we have

$$
\left[\begin{array}{cc}
D^{2}+\alpha_{1} D+\alpha_{2} & \alpha_{3} D+\alpha_{4} \\
\alpha_{5} D+\alpha_{6} & D^{2}+\alpha_{7} D+\alpha_{8}
\end{array}\right]\left[\begin{array}{c}
p_{2} \\
p_{2 A}
\end{array}\right]=\left[\begin{array}{l}
0 \\
0
\end{array}\right]
$$

Developing Eq. (A6), we have

$$
\begin{gathered}
p_{2}^{\prime \prime}+\alpha_{1} p_{2}^{\prime}+\alpha_{2} p_{2}+\alpha_{3} p_{2 A}^{\prime}+\alpha_{4} p_{2 A}=0 \\
\alpha_{5} p_{2}^{\prime}+\alpha_{6} p_{2}+p_{2 A}^{\prime \prime}+\alpha_{7} p_{2 A}^{\prime}+\alpha_{8} p_{2 A}=0
\end{gathered}
$$

Let

$$
p_{2}^{\prime}=\frac{d p_{2}}{d x}=y_{1}, p_{2 A}^{\prime}=\frac{d p_{2 A}}{d x}=y_{2}, p_{2}=y_{3}, p_{2 A}=y_{4}
$$

According to Eqs. (A7) and (A8), the new matrix between $\left\{y^{\prime}\right\}$ and $\{y\}$ is

$$
\left[\begin{array}{l}
y_{1}^{\prime} \\
y_{2}^{\prime} \\
y_{3}^{\prime} \\
y_{4}^{\prime}
\end{array}\right]=\left[\begin{array}{cccc}
-\alpha_{1} & -\alpha_{3} & -\alpha_{2} & -\alpha_{4} \\
-\alpha_{5} & -\alpha_{7} & -\alpha_{6} & -\alpha_{8} \\
1 & 0 & 0 & 0 \\
0 & 1 & 0 & 0
\end{array}\right]\left[\begin{array}{l}
y_{1} \\
y_{2} \\
y_{3} \\
y_{4}
\end{array}\right]
$$

which can be briefly expressed as

$$
\left\{y^{\prime}\right\}=[N]\{y\}
$$

Let

$$
\{y\}=[\Omega]\{\Gamma\}
$$

which is

$$
\left[\begin{array}{c}
d p_{2} / d x \\
d p_{2 A} / d x \\
p_{2} \\
p_{2 A}
\end{array}\right]=\left[\begin{array}{llll}
\Omega_{1,1} & \Omega_{1,2} & \Omega_{1,3} & \Omega_{1,4} \\
\Omega_{2,1} & \Omega_{2,2} & \Omega_{2,3} & \Omega_{2,4} \\
\Omega_{3,1} & \Omega_{3,2} & \Omega_{3,3} & \Omega_{3,4} \\
\Omega_{4,1} & \Omega_{4,2} & \Omega_{4,3} & \Omega_{4,4}
\end{array}\right]\left[\begin{array}{c}
\Gamma_{1} \\
\Gamma_{2} \\
\Gamma_{3} \\
\Gamma_{4}
\end{array}\right]
$$

$[\Omega]_{4 \times 4}$ is the model matrix formed by four sets of eigen vectors $\Omega_{4 x 1}$ of $[\mathrm{N}]_{4 x 4}$.

Substituting Eq. (A10) into Eq. (A19) and then multiplying $[\Omega]^{-1}$ by both sides, we have

$$
[\Omega]^{-1}[\Omega]\left\{\Gamma^{\prime}\right\}=[\Omega]^{-1}[\mathrm{~N}][\Omega]\{\Gamma\}
$$

Set 


$$
[\chi]=[\Omega]^{-1}[N][\Omega]=\left[\begin{array}{cccc}
\gamma_{1} & 0 & 0 & 0 \\
0 & \gamma_{2} & 0 & 0 \\
0 & 0 & \gamma_{3} & 0 \\
0 & 0 & 0 & \gamma_{4}
\end{array}\right]
$$

where $\gamma_{i}$ is the eigen value of [N].

Eq. (A10) can be thus rewritten as

$$
\left\{\Gamma^{\prime}\right\}=[\chi]\{\Gamma\}
$$

Obviously, Eq. (A11) is a decoupled equation. The related solution becomes

$$
\Gamma_{i}=k_{i} e^{\gamma_{i} x}
$$

Using Eqs. (A2), (A4), (A10), and (A12), the relationship of acoustic pressure and particle velocity becomes

$$
\left[\begin{array}{c}
p_{2}(x) \\
p_{2 A}(x) \\
\rho_{o} c_{o} u_{2}(x) \\
\rho_{o} c_{o} u_{2 A}(x)
\end{array}\right]=\left[\begin{array}{llll}
\mathrm{H}_{1,1} & \mathrm{H}_{1,2} & \mathrm{H}_{1,3} & \mathrm{H}_{1,4} \\
\mathrm{H}_{2,1} & \mathrm{H}_{2,2} & \mathrm{H}_{2,3} & \mathrm{H}_{2,4} \\
\mathrm{H}_{3,1} & \mathrm{H}_{3,2} & \mathrm{H}_{3,3} & \mathrm{H}_{3,4} \\
\mathrm{H}_{4,1} & \mathrm{H}_{4,2} & \mathrm{H}_{4,3} & \mathrm{H}_{4,4}
\end{array}\right]\left[\begin{array}{c}
k_{1} \\
k_{2} \\
k_{3} \\
k_{4}
\end{array}\right]
$$

Plugging $x=0$ and $x=L \mathrm{c}_{1}$ into Eq. (A13) yields

$$
\left[\begin{array}{c}
p_{2}(0) \\
p_{2 A}(0) \\
\rho_{o} c_{o} u_{2}(0) \\
\rho_{o} c_{o} u_{2 A}(0)
\end{array}\right]=[\mathrm{A}]\left[\begin{array}{c}
p_{2}\left(L_{C 1}\right) \\
p_{2 A}\left(L_{C 1}\right) \\
\rho_{o} c_{o} u_{2}\left(L_{C 1}\right) \\
\rho_{o} c_{o} u_{2 A}\left(L_{C 1}\right)
\end{array}\right]
$$

where

$$
[\mathrm{A}]=[\mathrm{H}(0)]\left[\mathrm{H}\left(L_{C 1}\right)\right]^{-1}
$$

To obtain the transform matrix between the inlet $(x=0)$ and the outlet $\left(x=L \mathrm{c}_{1}\right)$ of the inner tubes, the two boundary conditions for the outer tube at $x=0$ and $x=L \mathrm{c}_{1}$ are taken into calculation and listed below.

$$
\begin{aligned}
& \frac{p_{2 A}(0)}{-u_{2 A}(0)}=-j \rho_{o} c_{o} \cot \left(k L_{A 1}\right) \\
& \frac{p_{2 A}\left(L_{C 1}\right)}{u_{2 A}\left(L_{C 1}\right)}=-j \rho_{o} c_{o} \cot \left(k L_{B 1}\right)
\end{aligned}
$$

By substituting Eqs. (A15a, b) into Eq. (A14) and developing them, the transfer matrix becomes

$$
\left[\begin{array}{c}
p_{2} \\
\rho_{o} c_{o} u_{2}
\end{array}\right]=\left[\begin{array}{ll}
T P_{1,1} & T P_{1,2} \\
T P_{2,1} & T P_{2,2}
\end{array}\right]\left[\begin{array}{c}
p_{3} \\
\rho_{o} c_{o} u_{3}
\end{array}\right]
$$

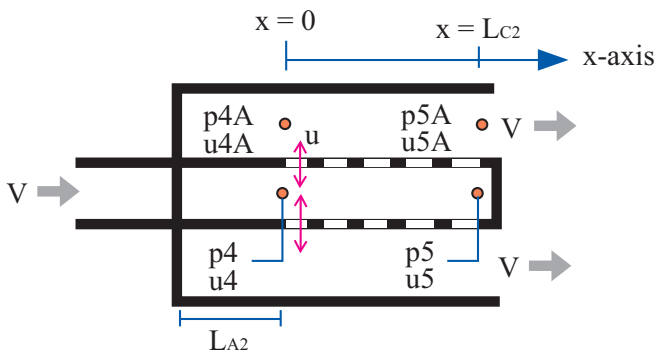

Fig. 13. Mechanism of an expanded perforated plug tube.

where

$$
p_{2}=p_{2}(0) ; u_{2}=u_{2}(0) ; p_{3}=p_{2}\left(L_{C 1}\right) ; u_{3}=u_{2}\left(L_{C 1}\right)(\mathrm{A} 16 \mathrm{~b})
$$

\section{APPENDIX B - Transfer Matrix of Expansion Perforated Duct}

As indicated in Fig. 13, the expansion perforated duct is composed of an inner perforated tube and an outer one-end opened duct. The continuity equations and momentum equations with respect to the inner and outer tubes at nodes 4 and $4 \mathrm{~A}$ are seen below [23].

\section{Inner tube:}

continuity equation

$$
V \frac{\partial \rho_{4}}{\partial x}+\rho_{o} \frac{\partial u_{4}}{\partial x}+\frac{4 \rho_{o}}{D_{1}} u+\frac{\partial \rho_{4 A}}{\partial t}=0
$$

momentum equation

$$
\rho_{o}\left(\frac{\partial}{\partial t}+V \frac{\partial}{\partial x}\right) u_{4}+\frac{\partial p_{4}}{\partial x}=0
$$

\section{Outer tube:}

continuity equation

$$
\rho_{o} \frac{\partial u_{4 A}}{\partial x}-\frac{4 D_{o} \rho_{o}}{D_{o}^{2}-D_{1}^{2}} u+\frac{\partial \rho_{4 A}}{\partial t}=0
$$

momentum equation

$$
\rho_{o} \frac{\partial u_{4 A}}{\partial t}+\frac{\partial p_{4 A}}{\partial x}=0
$$

Similarly, as derived in Eqs. (A1) (A9), we have

$$
\left[\begin{array}{cc}
D^{2}+\alpha_{11} D+\alpha_{21} & \alpha_{31} D+\alpha_{41} \\
\alpha_{51} D+\alpha_{61} & D^{2}+\alpha_{71} D+\alpha_{81}
\end{array}\right]\left[\begin{array}{c}
p_{4} \\
p_{44}
\end{array}\right]=\left[\begin{array}{l}
0 \\
0
\end{array}\right]
$$

Developing Eq. (B5), we obtain 


$$
\begin{gathered}
p_{4}^{\prime \prime}+\alpha_{11} p_{4}^{\prime}+\alpha_{21} p_{4}+\alpha_{31} p_{4 A}^{\prime}+\alpha_{41} p_{4 A}=0 \\
\alpha_{51} p_{4}^{\prime}+\alpha_{61} p_{4}+p_{4 A}^{\prime \prime}+\alpha_{71} p_{4 A}^{\prime}+\alpha_{81} p_{4 A}=0
\end{gathered}
$$

Let

$$
p_{4}^{\prime}=\frac{d p_{4}}{d x}=y_{11} ; p_{4 A}^{\prime}=\frac{d p_{4 A}}{d x}=y_{21} ; p_{4}=y_{31} ; p_{4 A}=y_{41}
$$

According to Eqs. (B6) and (B7), the new matrix between $\left\{y^{\prime}\right\}$ and $\{y\}$ is

$$
\left[\begin{array}{l}
y_{11}^{\prime} \\
y_{21}^{\prime} \\
y_{31}^{\prime} \\
y_{41}^{\prime}
\end{array}\right]=\left[\begin{array}{cccc}
-\alpha_{11} & -\alpha_{31} & -\alpha_{21} & -\alpha_{41} \\
-\alpha_{51} & -\alpha_{71} & -\alpha_{61} & -\alpha_{81} \\
1 & 0 & 0 & 0 \\
0 & 1 & 0 & 0
\end{array}\right]\left[\begin{array}{l}
y_{11} \\
y_{21} \\
y_{31} \\
y_{41}
\end{array}\right]
$$

which can be briefly expressed as

$$
\left\{y^{\prime}\right\}=[\Psi]\{y\}
$$

Let

$$
\{y\}=[\Omega \Omega]\{\Gamma \Gamma\}
$$

which is

$$
\left[\begin{array}{c}
d p_{4} / d x \\
d p_{4 A} / d x \\
p_{4} \\
p_{4 A}
\end{array}\right]=\left[\begin{array}{llll}
\Omega \Omega_{1,1} & \Omega \Omega_{1,2} & \Omega \Omega_{1,3} & \Omega \Omega_{1,4} \\
\Omega \Omega_{2,1} & \Omega \Omega_{2,2} & \Omega \Omega_{2,3} & \Omega \Omega_{2,4} \\
\Omega \Omega_{3,1} & \Omega \Omega_{3,2} & \Omega \Omega_{3,3} & \Omega \Omega_{3,4} \\
\Omega \Omega_{4,1} & \Omega \Omega_{4,2} & \Omega \Omega_{4,3} & \Omega \Omega_{4,4}
\end{array}\right]\left[\begin{array}{c}
\Gamma \Gamma_{1} \\
\Gamma \Gamma_{2} \\
\Gamma \Gamma_{3} \\
\Gamma \Gamma_{4}
\end{array}\right]
$$

$[\Omega \Omega]_{4 \times 4}$ is the model matrix formed by four sets of eigen vectors $\Omega \Omega_{4 x 1}$ of $[\Psi]_{4 \times 4}$.

Substituting Eq. (B9) into Eq. (B8) and then multiplying $[\Omega \Omega]^{-1}$ by both sides, we have

$$
[\Omega \Omega]^{-1}[\Omega \Omega]\left\{\Gamma \Gamma^{\prime}\right\}=[\Omega \Omega]^{-1}[\Psi][\Omega \Omega][\Gamma \Gamma]
$$

Set

$$
[\chi \chi]=[\Omega]^{-1}[\Psi][\Omega \Omega]=\left[\begin{array}{cccc}
\lambda \lambda_{1} & 0 & 0 & 0 \\
0 & \lambda \lambda_{2} & 0 & 0 \\
0 & 0 & \lambda \lambda_{3} & 0 \\
0 & 0 & 0 & \lambda \lambda_{4}
\end{array}\right](
$$

where $\lambda \lambda_{i}$ is the eigen value of $[\Psi]$.

Eq. (B9) can be thus rewritten as

$$
\left\{\Gamma \Gamma^{\prime}\right\}=[\chi \chi]\{\Gamma \Gamma\}
$$

Obviously, Eq. (B12) is a decoupled equation. The related solution is

$$
\Gamma \Gamma_{i}=f_{i} e^{\lambda_{i} \lambda x}
$$

Using Eqs. (B2), (B4), (B9) and (B13), the relationship of the acoustic pressure and the particle velocity is

$$
\left[\begin{array}{c}
p_{4}(x) \\
p_{4 A}(x) \\
\rho_{o} c_{o} u_{4}(x) \\
\rho_{o} c_{o} u_{4 A}(x)
\end{array}\right]=\left[\begin{array}{llll}
\mathrm{K}_{1,1} & \mathrm{~K}_{1,2} & \mathrm{~K}_{1,3} & \mathrm{~K}_{1,4} \\
\mathrm{~K}_{2,1} & \mathrm{~K}_{2,2} & \mathrm{~K}_{2,3} & \mathrm{~K}_{2,4} \\
\mathrm{~K}_{3,1} & \mathrm{~K}_{3,2} & \mathrm{~K}_{3,3} & \mathrm{~K}_{3,4} \\
\mathrm{~K}_{4,1} & \mathrm{~K}_{4,2} & \mathrm{~K}_{4,3} & \mathrm{~K}_{4,4}
\end{array}\right]\left[\begin{array}{c}
f_{1} \\
f_{2} \\
f_{3} \\
f_{4}
\end{array}\right]
$$

Plugging $x=0$ and $x=L_{\mathrm{C} 2}$ into Eq. (B14) yields

$$
\left[\begin{array}{c}
p_{4}(0) \\
p_{4 A}(0) \\
\rho_{o} c_{o} u_{4}(0) \\
\rho_{o} c_{o} u_{4 A}(0)
\end{array}\right]=[B]\left[\begin{array}{c}
p_{4}\left(L_{C 2}\right) \\
p_{4 A}\left(L_{C 2}\right) \\
\rho_{o} c_{o} u_{4}\left(L_{C 2}\right) \\
\rho_{o} c_{o} u_{4 A}\left(L_{C 2}\right)
\end{array}\right]
$$

where

$$
[B]=[\mathrm{K}(0)]\left[\mathrm{K}\left(L_{C 2}\right)\right]^{-1}
$$

To obtain the transform matrix between inlet $(x=0)$ and outlet $\left(x=L_{\mathrm{C} 2}\right)$ of the inner tubes, two boundary conditions for the outer tube at $x=0$ and $x=L_{\mathrm{C} 2}$ are calculated and listed below.

$$
\begin{gathered}
\frac{p_{4 A}(0)}{-u_{4 A}(0)}=-j \rho_{o} c_{o} \cot \left(k L_{A 2}\right) \\
\frac{p_{4}\left(L_{C 2}\right)}{u_{4}\left(L_{C 2}\right)}=-j \rho_{o} c_{o} \cot (0)
\end{gathered}
$$

By substituting Eq. (B16) for Eq. (B16) and developing them, the transfer matrix is

$$
\left[\begin{array}{c}
p_{4} \\
\rho_{o} c_{o} u_{4}
\end{array}\right]=\left[\begin{array}{cc}
T P E_{1,1} & T P E_{1,2} \\
T P E_{2,1} & T P E_{2,2}
\end{array}\right]\left[\begin{array}{c}
p_{5 A} \\
\rho_{o} c_{o} u_{5 A}
\end{array}\right]
$$

where

$$
p_{4}=p_{4}(0) ; u_{4}=u_{4}(0) ; p_{5 A}=p_{4 A}\left(L_{C 2}\right) ; u_{5 A}=u_{4 A}\left(L_{C 2}\right)(\mathrm{B} 17 \mathrm{~b})
$$

\section{APPENDIX C - Transfer Matrix of Contracted Perforated Duct}

As indicated in Fig. 14, the contracted perforated duct is composed of an inner perforated tube and an outer contracted tube. The continuity equations and momentum equations with 


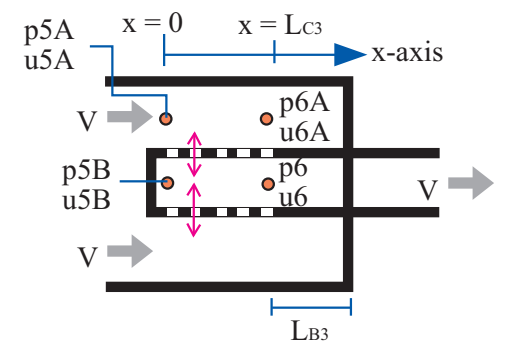

Fig. 14. Mechanism of a contracted perforated plug tube.

respect to the inner and outer tubes at nodes $5 \mathrm{~B}$ and $5 \mathrm{~A}$ are as follows [23].

\section{Inner tube:}

continuity equation

$$
V \frac{\partial \rho_{5 B}}{\partial x}+\rho_{o} \frac{\partial u_{5 B}}{\partial x}+\frac{4 \rho_{o}}{D_{1}} u+\frac{\partial \rho_{5 A}}{\partial t}=0
$$

momentum equation

$$
\rho_{o}\left(\frac{\partial}{\partial t}+V \frac{\partial}{\partial x}\right) u_{5 B}+\frac{\partial p_{5 B}}{\partial x}=0
$$

\section{Outer tube:}

continuity equation

$$
\rho_{o} \frac{\partial u_{5 A}}{\partial x}-\frac{4 D_{o} \rho_{o}}{D_{o}^{2}-D_{1}^{2}} u+\frac{\partial \rho_{5 A}}{\partial t}=0
$$

momentum equation

$$
\rho_{o} \frac{\partial u_{5 A}}{\partial t}+\frac{\partial p_{5 A}}{\partial x}=0
$$

Likewise, as derived in Eqs. (A1) (A8), we have

$$
\left[\begin{array}{cc}
D^{2}+\alpha_{12} D+\alpha_{22} & \alpha_{32} D+\alpha_{42} \\
\alpha_{52} D+\alpha_{62} & D^{2}+\alpha_{72} D+\alpha_{82}
\end{array}\right]\left[\begin{array}{l}
p_{5 B} \\
p_{5 A}
\end{array}\right]=\left[\begin{array}{l}
0 \\
0
\end{array}\right]
$$

Developing Eq. (C5) becomes

$$
\begin{aligned}
& p_{5 B}^{\prime \prime}+\alpha_{12} p_{5 B}^{\prime}+\alpha_{22} p_{5 B}+\alpha_{32} p_{5 A}^{\prime}+\alpha_{42} p_{5 A}=0 \\
& \alpha_{52} p_{5 B}^{\prime}+\alpha_{62} p_{5 B}+p_{5 A}^{\prime \prime}+\alpha_{72} p_{5 A}^{\prime}+\alpha_{82} p_{5 A}=0
\end{aligned}
$$

Let

$p_{5 B}^{\prime}=\frac{d p_{5 B}}{d x}=y_{12} ; p_{5 A}^{\prime}=\frac{d p_{5 A}}{d x}=y_{22} ; p_{5 B}=y_{32} ; p_{5 A}=y_{42}$
According to Eqs. (C6) and (C7), the new matrix between $\left\{y^{\prime}\right\}$ and $\{y\}$ is

$$
\left[\begin{array}{l}
y_{12}^{\prime} \\
y_{22}^{\prime} \\
y_{32}^{\prime} \\
y_{42}^{\prime}
\end{array}\right]=\left[\begin{array}{cccc}
-\alpha_{12} & -\alpha_{32} & -\alpha_{22} & -\alpha_{42} \\
-\alpha_{52} & -\alpha_{72} & -\alpha_{62} & -\alpha_{82} \\
1 & 0 & 0 & 0 \\
0 & 1 & 0 & 0
\end{array}\right]\left[\begin{array}{l}
y_{12} \\
y_{22} \\
y_{32} \\
y_{42}
\end{array}\right]
$$

which can be briefly expressed as

$$
\left\{y^{\prime}\right\}=[\Psi \Psi]\{y\}
$$

Let

$$
\{y\}=[\Omega \Omega \Omega]\{\Gamma \Gamma \Gamma\}
$$

which is

$\left[\begin{array}{c}d p_{5 B} / d x \\ d p_{5 A} / d x \\ p_{5 B} \\ p_{5 A}\end{array}\right]=\left[\begin{array}{llll}\Omega \Omega \Omega_{1,1} & \Omega \Omega \Omega_{1,2} & \Omega \Omega \Omega_{1,3} & \Omega \Omega \Omega_{1,4} \\ \Omega \Omega \Omega_{2,1} & \Omega \Omega \Omega_{2,2} & \Omega \Omega \Omega_{2,3} & \Omega \Omega \Omega_{2,4} \\ \Omega \Omega \Omega_{3,1} & \Omega \Omega \Omega_{3,2} & \Omega \Omega \Omega_{3,3} & \Omega \Omega \Omega_{3,4} \\ \Omega \Omega \Omega_{4,1} & \Omega \Omega \Omega_{4,2} & \Omega \Omega \Omega_{4,3} & \Omega \Omega \Omega_{4,4}\end{array}\right]\left[\begin{array}{l}\Gamma \Gamma \Gamma_{1} \\ \Gamma \Gamma \Gamma_{2} \\ \Gamma \Gamma \Gamma_{3} \\ \Gamma \Gamma \Gamma_{4}\end{array}\right]$

$[\Omega \Omega \Omega]_{4 x 4}$ is the model matrix formed by four sets of eigen vectors $\Omega \Omega \Omega_{4 x 1}$ of $[\Psi \Psi]_{4 x 4}$

Substituting Eq. (C9) for Eq. (C8) and then multiplying $[\Omega \Omega \Omega]^{-1}$ by both sides, we have

$[\Omega \Omega \Omega]^{-1}[\Omega \Omega \Omega]\left\{\Gamma \Gamma \Gamma^{\prime}\right\}=[\Omega \Omega \Omega]^{-1}[\Psi \Psi][\Omega \Omega \Omega]\{\Gamma \Gamma \Gamma\}(\mathrm{C} 10)$

Set

$[\chi \chi \chi]=[\Omega \Omega \Omega]^{-1}[\Psi \Psi][\Omega \Omega \Omega]=\left[\begin{array}{cccc}\lambda \lambda \lambda_{1} & 0 & 0 & 0 \\ 0 & \lambda \lambda \lambda_{2} & 0 & 0 \\ 0 & 0 & \lambda \lambda \lambda_{3} & 0 \\ 0 & 0 & 0 & \lambda \lambda \lambda_{4}\end{array}\right]$

where $\lambda \lambda \lambda_{i}$ is the eigen value of [ $\left.\Psi \Psi\right]$.

Eq. (C9) can thus be rewritten as

$$
\left\{\Gamma \Gamma \Gamma^{\prime}\right\}=[\chi \chi \chi]\{\Gamma \Gamma \Gamma\}
$$

Obviously, Eq. (C12) is a decoupled equation. The related solution becomes

$$
\Gamma \Gamma \Gamma_{i}=f f_{i} e^{\lambda \lambda \lambda_{i} x}
$$

Using Eqs. (C2), (C4), (C7) and (C13), the relationship of the acoustic pressure and the particle velocity becomes 


$$
\left[\begin{array}{c}
p_{5 B}(x) \\
p_{5 A}(x) \\
\rho_{o} c_{o} u_{5 B}(x) \\
\rho_{o} c_{o} u_{5 A}(x)
\end{array}\right]=\left[\begin{array}{llll}
\mathrm{KK}_{1,1} & \mathrm{KK}_{1,2} & \mathrm{KK}_{1,3} & \mathrm{KK}_{1,4} \\
\mathrm{KK}_{2,1} & \mathrm{KK}_{2,2} & \mathrm{KK}_{2,3} & \mathrm{KK}_{2,4} \\
\mathrm{KK}_{3,1} & \mathrm{KK}_{3,2} & \mathrm{KK}_{3,3} & \mathrm{KK}_{3,4} \\
\mathrm{KK}_{4,1} & \mathrm{KK}_{4,2} & \mathrm{KK}_{4,3} & \mathrm{KK}_{4,4}
\end{array}\right]\left[\begin{array}{c}
f f_{1} \\
f f_{2} \\
f f_{3} \\
f f_{4}
\end{array}\right]
$$

Plugging $x=0$ and $x=L_{\mathrm{C} 3}$ into Eq. (C14) yields

$$
\left[\begin{array}{c}
p_{5 B}(0) \\
p_{5 A}(0) \\
\rho_{o} c_{o} u_{5 B}(0) \\
\rho_{o} c_{o} u_{5 A}(0)
\end{array}\right]=[B B]\left[\begin{array}{c}
p_{5 B}\left(L_{C 3}\right) \\
p_{5 A}\left(L_{C 3}\right) \\
\rho_{o} c_{o} u_{5 B}\left(L_{C 3}\right) \\
\rho_{o} c_{o} u_{5 A}\left(L_{C 3}\right)
\end{array}\right]
$$

where

$$
[B B]=[\mathrm{KK}(0)]\left[\mathrm{KK}\left(L_{C 3}\right)\right]^{-1}
$$

To obtain the transform matrix between inlet $(x=0)$ and outlet $\left(x=L_{\mathrm{C} 3}\right)$ of the inner tubes, two boundary conditions for the outer tube at $x=0$ and $x=L_{\mathrm{C} 3}$ are calculated and listed below.

$$
\begin{gathered}
\frac{p_{5 B}(0)}{-u_{5 B}(0)}=-j \rho_{o} c_{o} \cot (0) \\
\frac{p_{5 A}\left(L_{C 3}\right)}{u_{5 A}\left(L_{C 3}\right)}=-j \rho_{o} c_{o} \cot \left(k L_{B 3}\right)
\end{gathered}
$$

By substituting Eqs. (C16) into Eq. (C15) and developing them, the transfer matrix is

$$
\left[\begin{array}{c}
p_{5 A} \\
\rho_{o} c_{o} u_{5 A}
\end{array}\right]=\left[\begin{array}{ll}
T P C_{1,1} & T P C_{1,2} \\
T P C_{2,1} & T P C_{2,2}
\end{array}\right]\left[\begin{array}{c}
p_{6} \\
\rho_{o} c_{o} u_{6}
\end{array}\right]
$$

where

$$
p_{5 A}=p_{5 A}(0) ; u_{5 A}=u_{5 A}(0) ; p_{6}=p_{5 B}\left(L_{C 3}\right) ; u_{6}=u_{5 B}\left(L_{C 3}\right)
$$

\section{REFERENCES}

1. Chang, Y. C., Yeh, L. J., Chiu, M. C., and Lai, G. J., "Shape optimization on constrained single-layer sound absorber by using GA method and mathematical gradient methods," Journal of Sound and Vibration, Vol. 286, No. 4-5, pp. 941-961 (2005).

2. Chiu, M. C., "Shape optimization of double-chamber side mufflers with extended tube by using four-pole matrix and simulated annealing method," Journal of Mechanics, Vol. 24, pp. 31-43 (2008).

3. Chiu, M. C. and Chang, Y. C., "Numerical studies on venting system with multi-chamber perforated mufflers by GA optimization," Applied Acoustics, Vol. 69, No. 11, pp. 1017-1037 (2008).

4. Chiu, M. C., Chang, Y. C., and Yeh, L. J., "Numerical assessment of optimal one-chamber perforated mufflers by using GA method," Material
Science Forum, Vol. 594, pp. 368-376 (2008).

5. Glover, F., "Heuristics for inter programming using surrogate constraints," Decision Sciences, Vol. 8, No. 1, pp. 156-166 (1977).

6. Jayaraman, K. and Yam, K., "Decoupling approach to modeling perforated tube muffler component," Journal of the Acoustical Society of America, Vol. 69, No. 2, pp. 390-396 (1981).

7. Kar, T. and Munjal, M. L., "Generalized analysis of a muffler with any number of interacting ducts," Journal of Sound and Vibration, Vol. 285, pp. 585-596 (2005).

8. Kirkpatrick, S., Gelatt, C. D. and Vecchi, M. P., "Optimization by simulated annealing," Science, Vol. 220, pp. 671-680 (1983).

9. Laurence, W., Integer Programming, John Wiley \& Sons, New York (1998).

10. Lee, L. and Selamet, A., "Impact of perforation impedance on the transmission loss of reactive and dissipative silencers," Journal of the Acoustical Society of America, Vol. 120, No. 6, pp. 3706-3713 (2006).

11. Metropolis, A., Rosenbluth, W., Rosenbluth, M. N., Teller, H., and Teller, E., "Equation of static calculations by fast computing machines," Journal of Chemical Physics, Vol. 21, pp. 1087-1092 (1953).

12. Munjal, M. L., Acoustics of Ducts and Mufflers with Application to Exhaust and Ventilation System Design, John Wiley \& Sons, New York (1987).

13. Munjal, M. L., Krishnan, K., and Reddy, M. M., "Flow-acoustic perforated element mufflers with application to design," Noise Control Engineering Journal, Vol. 40, No. 1, pp. 159-167 (1993).

14. Munjal, M. L., Rao, K. N., and Sahasrabudhe, A. D., "Aeroacoustic analysis of perforated muffler components," Journal of Sound and Vibration, Vol. 114, No. 2, pp. 173-188 (1987).

15. Nolle, L., Armstrong, D. A., Hopgood, A. A., and Ware, J. A., "Simulated annealing and genetic algorithms applied to finishing mill optimization for hot rolling of wide steel strip," International of Knowledge-Based Intelligent Engineering System, Vol. 6, pp. 104-111 (2002).

16. Peat, K. S., "A numerical decoupling analysis of perforated pipe silencer elements," Journal of Sound and Vibration, Vol. 123, No. 2, pp. 199-212 (1988).

17. Rao, K. N. and Munjal, M. L., "A generalized decoupling method for analyzing perforated element mufflers," Nelson Acoustics Conference, Madison (1984).

18. Rardin, R. L., Optimization in Operations Research, Prentice Hall, New Jersey (1998).

19. Reklaitis, G. V., Ravindran, A., and Ragsdell, K. M., Engineering Optimization: Method and Applications, Wiley, New York (1984).

20. Sohei, N., Tsuyoshi, N., and Takashi, Y., "Acoustic analysis of elliptical muffler chamber having a perforated pipe," Journal of Sound and Vibration, Vol. 297, pp. 761-773 (2006).

21. Sullivan, J. W., "A method of modeling perforated tube muffler components I: theory," Journal of the Acoustical Society of America, Vol. 66, pp. 772-778 (1979).

22. Sullivan, J. W., "A method of modeling perforated tube muffler components II: applications," Journal of the Acoustical Society of America, Vol. 66, pp. 779-788 (1979).

23. Sullivan, J. W. and Crocker, M. J., "Analysis of concentric tube resonators having unpartitioned cavities," Journal of the Acoustical Society of America, Vol. 64, pp. 207-215 (1978).

24. Thawani, P. T. and Jayaraman, K., "Modeling and applications of straightthrough resonators," Journal of the Acoustical Society of America, Vol. 73 , No. 4, pp. 1387-1389 (1983).

25. Vanderplaats, N. G., Numerical optimization techniques for engineering design: with applications, McGraw-Hill, New York (1984).

26. Weeber, K., Ratnajeevan, S., and Hoole, H., "Geometric parametrization and constrained optimization techniques in the design of salient pole synchro nous machines," IEEE Transaction on Magnetics, Vol. 28, No. 4, pp. 1948-1960 (1992).

27. Yeh, L. J., Chang, Y. C., Chiu, M. C., and Lai, G. J., "GA optimization on multi-segments muffler under space constraints," Applied Acoustics, Vol. 65 , No. 5, pp. 521-543 (2004). 